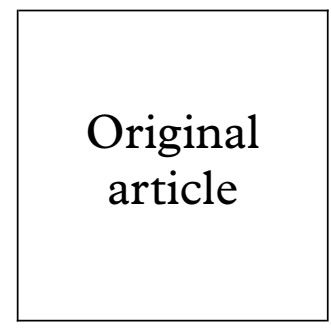

\title{
Pooling of urine specimens for PCR testing: a cost saving strategy for Chlamydia trachomatis control programmes
}

\author{
Rosanna W Peeling, Baldwin Toye, Peter Jessamine, Ian Gemmill
}

\begin{abstract}
Objectives: To evaluate pooling of first catch urine (FCU) specimens as a cost effective strategy for chlamydia testing.

Methods: Mock specimens were pooled with and without dilution to determine optimal pool size and ease of work flow. The performance of the Amplicor Chlamydia trachomatis PCR assay on pooled specimens was compared with individual testing using 370 FCU specimens from asymptomatic men presenting to an STD clinic. Cost savings associated with pooling were estimated. Results: Using mock specimens, the sensitivity and specificity of the Amplicor PCR assay were not affected by pool sizes of two and five, but at a pool size of 10 decreased sensitivity due to inhibition was observed in one of five mock pools when the pooling method which involved no dilution was used. Archived FCU specimens from a study of 370 asymptomatic men were combined consecutively into 74 pools of five and tested by PCR. Of the 18 pools that contained positive specimens, 17 were PCR positive. Compared with testing FCU specimens individually, pooling resulted in a sensitivity of $95 \%$, specificity of $100 \%$, and a cost savings of $57 \%$ based on reduced number of tests required.
\end{abstract}

Conclusion: Depending on the prevalence of infection, pooling of FCU specimens for PCR testing may result in cost savings compared with testing specimens individually. Further evaluations to validate this strategy using fresh FCU specimens are needed.

(Sex Transm Inf 1998;74:66-70)

Keywords: urine specimens; polymerase chain reaction; cost; Chlamydia trachomatis

\section{Introduction}

Genital chlamydial infection is the leading cause of bacterial sexually transmitted diseases (STDs). The World Health Organisation estimated that there were 89 million new cases of genital chlamydial infections worldwide in 1995. ${ }^{1}$ In the United States, there are an estimated four million new cases each year and 50000 women annually become infertile as a result of their infection. ${ }^{2}$

A hallmark of human chlamydial infection is that $50-70 \%$ of genital infections are asymptomatic. Asymptomatically infected individuals act as a reservoir which maintains the prevalence of infection in a population unless individuals at risk of infection are targeted for screening. Targeted screening for cervical chlamydial infections has recently been shown to be effective in decreasing the incidence of symptomatic pelvic inflammatory disease (PID). ${ }^{3}$ As individuals with bacterial STDs, including chlamydial infections, are at increased risk of acquisition of HIV, control of genital chlamydial infection could potentially have a major impact on HIV transmission. ${ }^{4}$ A recent study in Tanzania showed that strengthening STD control through education, access to diagnosis and treatment resulted in a $42 \%$ reduction in the incidence of HIV infection over a 2 year period. ${ }^{5}$ Targeted screening for genital chlamydial infections in high risk populations should therefore be a priority for STD/HIV control programmes.

Enzyme immunoassay (EIA) is the diagnostic method most commonly used for the laboratory diagnosis of chlamydial infections. EIA has a lower detection limit of 10000 elementary bodies and thus lacks sensitivity required for a screening assay, especially in asymptomatic men. ${ }^{6-8}$ Nucleic acid based amplification assays using polymerase chain reaction (PCR), ligase chain reaction (LCR), or transcription mediated amplification technology are now commercially available. These assays have a lower detection limit of one to 10 elementary bodies and specificities comparable with culture..$^{8-11}$ They also offer all the advantages of non-culture tests in terms of specimen transport, batching, and rapid processing time of approximately 4 hours and, in addition, automation for some assays. The improved sensitivity of these assays allows the use of non-invasive specimens such as first catch urine (FCU) specimens. PCR tests using FCU specimens have been shown to have sensitivities ranging from $87 \%$ to $97 \%$ for men and $82 \%$ to $93 \%$ for women with specificities of $98-100 \%$ for both men and women. ${ }^{12-17}$ Studies on LCR assays using FCU specimens report sensitivities of $77-94 \%$ for men and $78-96 \%$ for women with specificities of $97-100 \%$ for men and $99-100 \%$ for women. ${ }^{18-22}$ Non-invasive specimens such as FCU are more acceptable to patients than urethral swabs and also eliminate potential sampling bias which has been observed with urethral swab samples. The ease of collection and transport of urine specimens makes large scale screening of targeted populations feasible in settings outside physicians' offices. 
A major drawback of utilising molecular technology in control programmes for testing or screening is the high cost of these laboratory tests. In particular, with the gradual decline in the incidence of genital chlamydial infections in countries where control programmes have been in place, a more cost effective means of utilising this new technology for disease eradication is increasingly necessary. ${ }^{8}$ One such strategy is to pool urine specimens for testing. Pooling of sera for seroprevalence studies of HIV has been shown to be cost effective. ${ }^{23}$ As nucleic acid based amplification assays are performed on the urine pellets after a centrifugation step, specimens can be pooled with minimal dilution effect. Specimens from pools which show a positive test result would then be tested individually. In this study, we determined the effect of different urine pool sizes on the sensitivity and specificity of PCR assay using mock specimens as well as archived urine specimens from a study of asymptomatic men attending an STD clinic. ${ }^{15}$ We also validated a formula used to estimate the number of pools testing positive and calculated potential savings as a result of pooling.

\section{Methods}

The optimal urine pool size and the effect of pooling on the sensitivity and specificity of PCR assay were determined by testing mock specimens as well as archived FCU specimens.

MOCK SPECIMENS

We explored two methods of pooling using mock specimens since there were advantages and disadvantages with each method. The first pooling method was designed to minimise any dilution effect by solubilising all the urine pellets in the pool using a single specimen volume of lysis buffer. However, reprocessing of FCU specimens would be required once a positive pool was identified, before the specimens in the pool could be tested individually. The second pooling method consisted of combining aliquots of solubilised urine pellets to make up the pooled sample. Inherent in this method of pooling was a dilution effect, but it allowed for an easier work flow in that once a positive pool was identified each specimen in the pool could be individually tested without having to process FCU specimens again.

FCU specimens had been stored for up to 6 months at $-20^{\circ} \mathrm{C}$ after an aliquot was taken for PCR testing using the Amplicor Chlamydia trachomatis PCR assay with a microwell plate detection method (Roche Diagnostics Systems, Branchburg, NJ, USA). The FCU specimens were thawed and a known PCR positive FCU specimen was pooled with one, four, and nine PCR negative FCU specimens to make up pool sizes of two, five, and 10 respectively. For each pool size, five different pools were made up using different FCU specimens. Mocked pools were prepared with reagents from the Amplicor Chlamydia trachomatis PCR assay using two methods which involved a common processing protocol until the resuspension of the urine pellet. The common processing protocol involves combining $500 \mu \mathrm{l}$ of each FCU specimen with $500 \mu$ l of urine wash buffer. Each mixture was vortexed, incubated at $37^{\circ} \mathrm{C}$ for 15 minutes, mixed again by vortexing, and centrifuged at $13000 \mathrm{~g}$ for 5 minutes. After the supernatants were discarded, two different methods of pooling were used. In the first method (no dilution), $250 \mu 1$ of lysis buffer was used to resuspend the first urine pellet within each pool. The resulting suspension was transferred sequentially to solubilise all other pellets within the pool before being left at room temperature for 15 minutes. The suspension was then mixed with $250 \mu \mathrm{l}$ of urine diluent by vortexing. This mixture was centrifuged at 13000 $g$ for 10 minutes and $50 \mu \mathrm{l}$ of the supernatant was used in the Amplicor Chlamydia trachomatis PCR assay according to the manufacturer's directions. The second method of pooling (with dilution) involved using $250 \mu \mathrm{l}$ of urine diluent to resuspend each urine pellet. Each mixture was left at room temperature for 15 minutes, and then mixed with $250 \mu$ of urine diluent by vortexing. The mixture was centrifuged at $13000 \mathrm{~g}$ for 10 minutes and $50 \mu \mathrm{l}$ of each supernatant in the pool was used to make up a pooled suspension, of which $50 \mu$ l were used for testing in the Amplicor Chlamydia trachomatis PCR assay according to manufacturer's directions. All supernatants were stored at $4^{\circ} \mathrm{C}$ for further testing if necessary.

\section{ARCHIVED SPECIMENS}

Archived FCU specimens from a study of asymptomatic men attending an STD clinic were used. ${ }^{15}$ These urine specimens had previously been tested individually by PCR using the Amplicor Chlamydia trachomatis PCR assay. Positive specimens had been confirmed by a positive urethral swab culture from the same patient or by a second PCR assay using primers directed at the major outer membrane protein gene of $C$ trachomatis. The processed FCU specimens were stored in specimen diluent at $-20^{\circ} \mathrm{C}$ until pooled. The stored specimens were thawed and pooled in groups of five by combining $50 \mu \mathrm{l}$ aliquots of each stored specimen from five consecutive FCU specimens to yield a total volume of $250 \mu \mathrm{l}$ per pool. From each pool, $50 \mu \mathrm{l}$ were then tested using the Cobas Amplicor Chlamydia trachomatis PCR assay (Roche Diagnostic Systems, Branchburg, NJ, USA) according to manufacturer's instructions. The Cobas assay is an automated version of the Amplicor Chlamydia trachomatis PCR assay. DNA amplification processes such as PCR may be inhibited by substances present in the clinical specimens. In order to ensure the validity of a negative result internal control (IC) DNA provided with this assay was added to the PCR master mix. This IC DNA contains primer binding regions identical to those of the $C$ trachomatis target sequence but with a unique internal probe binding region. This allows simultaneous amplification of both $C$ trachomatis target DNA, if present in the clinical specimen, and IC target DNA followed by selective detection of either amplicon. Failure to detect the IC target DNA after amplification would indicate that inhibition of PCR had occurred. The individual performing the PCR 
Table 1 Estimated cost savings using the pooling strategy for 1000 FCU specimens

\begin{tabular}{llll}
\hline \multirow{2}{*}{$\begin{array}{l}\text { Estimated disease } \\
\text { prevalence }\end{array}$} & Size of pool & No of tests required & \\
\cline { 3 - 4 } & Pools + individual tests & Cost savingst \\
\hline $20 \%$ & 2 & $500+360$ & $14 \%$ \\
$10 \%$ & 5 & $200+670$ & $13 \%$ \\
$5 \%$ & 2 & $500+190$ & $31 \%$ \\
& 5 & $200+410$ & $39 \%$ \\
& 5 & $200+100$ & $50 \%$ \\
\hline
\end{tabular}

${ }^{\star}$ The number of individual tests required was calculated using the formula of Raboud et $a l^{23}$ to estimate the number of pools testing positive.

The probability of a pool testing positive is given by the formula: $1-(1-P)^{n}$, where $P=$ the probability of an individual specimen testing positive and $n=$ the size of the pool.

t\% cost savings calculated as percentage reduction in no of tests required using pooling strategy compared with testing each FCU specimen individually.

assays on the pooled specimens was unaware of the original PCR results.

ESTIMATION OF POTENTIAL COST SAVINGS

The cost savings associated with pooling is dependent on the prevalence of infection, the size of pools, and the extent of clustering of positive specimens in the study population. A formula for estimating the number of positive pools was adapted from a formula used for pooling sera for seroprevalence studies of HIV infection. ${ }^{23}$ If each specimen in the pool has no effect on the outcome of the test for other specimens in the pool, then the probability of a pool testing positive is given by the formula:

$$
1-(1-P)^{n}
$$

where $\mathrm{P}=$ the probability of an individual specimen testing positive and $n=$ the size of the pool.

Since the cost of testing varies greatly for different laboratories according to the cost of test kits and labour, there was no attempt made to estimate comprehensive costs for testing. Instead, for these estimations to have wider applicability, we calculated the potential cost savings based only on the percentage reduction of the number of tests associated with the pooling strategy using the above formula.

\section{Results}

The mock specimens with pool sizes of two and five were all positive by PCR using both pooling methods. There were no false positives. However, one of the five pools of 10 tested negative by the first pooling method (no dilution method) because of inhibition of the PCR reaction. As a result, a pool size of five and the second pooling method were chosen for the testing of the archived urine specimens.

There were 370 archived FCU specimens available for pooling, which included 19 specimens previously found to be positive by PCR. Of the 74 pools, 18 contained PCR positive specimens (one pool contained two individual FCU specimens which were positive). Seventeen of these 18 pools tested positive by PCR. When the specimens in the 17 positive pools were tested individually, 18 of the 19 previously PCR positive specimens were identified. None of the 56 pools which contained only PCR negative specimens were positive. All pools negative for $C$ trachomatis DNA had a positive internal control indicating the absence of inhibition. The sensitivity of the pooling strategy was therefore $95 \%$ and the specificity was $100 \%$ compared with individual testing.
The pool which tested negative but contained a positive specimen was retested on two separate occasions and found to be positive on only one of them. As a result, this pool was made up again by repooling the individual FCU specimens which comprised the pool. PCR testing of this repeat pool was performed on three separate occasions and found again to be positive on only one of them. To investigate this further, this individual PCR positive pooled negative FCU specimen was diluted 1:5 and 1:10 using specimen diluent. PCR testing was positive on the undiluted specimen, equivocal (that is, "grey zone", absorbance of 0.217 ) on the $1: 5$ dilution, and negative on the $1: 10$ dilution.

Table 1 demonstrates the effect of disease prevalence and pool size on the estimated number of positive pools and the cost savings based on the reduction in the number of tests required. Using a pool size of five and the results from the 370 archived FCU specimens from asymptomatic men (prevalence of $5.1 \%$ ), 74 pools were tested and subsequently, individual FCU specimens from 17 of these pools would require further testing. This resulted in a total of 159 tests being performed for screening these men compared with 370 . This pooling strategy would have resulted in a $57 \%$ reduction in the number of tests performed, consistent with the estimate in table 1 using the formula of Raboud et al. ${ }^{23}$

\section{Discussion}

The use of non-invasive specimens for molecular diagnosis and screening for genital chlamydial infections is expected to have a major impact on the effectiveness of control programmes. However, this approach is not widely accessible at present as the cost of molecular tests are prohibitive for chlamydia control programmes in most countries. The strategy described here where urine specimens were pooled for testing demonstrated potential savings of up to $57 \%$ on the cost of test kits alone.

The results of this study showed that pooling did not have any effect on the specificity of the chlamydia PCR assay. A potential limitation of the pooling strategy was a possible loss of sensitivity due to either inhibition of the PCR reaction or a dilution effect, depending on the method of pooling used. Urine specimens are uniquely suited to pooling as testing can be performed on the urine pellet after centrifugation without any dilution effect, which was the basis for the first pooling method. However, inhibition of the PCR reaction was observed in one of five mock pools when the pool size reached 10, using this method of pooling.

False negative PCR or LCR results have been encountered in many evaluations of DNA amplification based techniques using culture as a gold standard. A survey of recent studies showed that $3-14 \%$ of FCU specimens from men and $4-15 \%$ of FCU specimens from women were negative by PCR or LCR when the corresponding urethral or cervical swab cultures were positive (table 2). The frequency of false negative PCR or LCR FCU specimens 
encountered in these studies did not appear to be related to sex or to the prevalence of infection. Although there may be multiple reasons for these discrepant results, such as inhibitors in the FCU, low bacterial burden, or lack of concurrent urethral infection in women with cervical infection, a large number of these inhibitory reactions were resolved by phenol chloroform extraction or by dilution of the sample before retesting, suggesting that there might have been inhibitory substances in the majority of these false negative FCU specimens. Verkooyen et al studied methods of reducing PCR inhibitors in cervical specimens and recommended a 10 -fold dilution of the specimen followed by heat treatment at $95^{\circ} \mathrm{C}$ for 10 minutes as the most effective means of preventing PCR inhibition. ${ }^{24}$ Although inhibitors in FCU specimens may be different from those in cervical swabs, dilution of FCU has been used successfully to resolve discrepant results with urethral culture. If the second method of pooling were used, the dilution resulting from pooling may serve to offset the loss of sensitivity due to inhibitors in the specimens. Thus, this pooling strategy may have dual advantages of cost saving as well as diluting out inhibitory substances before testing.

A major advantage of the second pooling method is that there is no need to reprocess FCU specimens once positive pools are identified. Although loss of sensitivity was not observed for any of the mock specimens as a result of the dilution, a false negative result was observed in one of the pools of archived FCU specimens. Since the sensitivity of the PCR test is such that less than $5 \%$ of PCR positive specimens have absorbance values of less than 3.0, this dilution effect is not expected to have an adverse effect on test sensitivity for the majority of specimens ${ }^{11}$ (unpublished data from our laboratory).

This study was carried out on previously frozen FCU specimens. Although inhibitory substances in the specimens are not significantly affected by freezing and thawing, the effects of inhibitors and dilution on the sensitivity and specificity of this pooling strategy should be confirmed in large prospective studies using fresh FCU specimens. ${ }^{24}$

The effectiveness of the pooling strategy is dependent on the prevalence of infection, the pool size used, and the clustering of positive specimens within the population tested. As shown in table 1 , pooling is not cost effective when disease prevalence is $20 \%$ or greater; however, as disease prevalence decreases, the cost savings associated with this pooling strategy increases, resulting in a potential saving of more than $50 \%$ when the disease prevalence is $5 \%$. In countries where chlamydia control programmes have been in place in recent years, the prevalence of chlamydial infection has been declining, thus increasing the cost of screening. Substantial savings on screening programmes may be realised utilising this pooling strategy. Potential cost savings are also dependent on the clustering of positive specimens - that is, distribution of positive specimens in the specimen pools tested. The results of the archived FCU from the asymptomatic men study validated the use of the formula of Raboud et al for estimation of potential savings based on prevalence of infection in the population tested and the pool size. $^{23}$

This pooling strategy is most useful for large scale studies to study the epidemiology of these infections and for population based screening programmes. It may also be a cost effective means of evaluating the impact of control programmes and of monitoring the prevalence of infection in target populations to determine if screening is cost effective. The use of pooling for routine testing may be desirable in laboratories which process a large number of specimens. However, this will depend on the prevalence of infection in the population tested and the acceptability of a delay in reporting of results to allow for testing of individual specimens in pools that test positive. In resource limited settings, pooling may be useful for large epidemiological surveys as well as a means of utilising molecular technology to validate algorithms for syndromic management.

Given the enormous costs of long term reproductive sequelae in women with undetected genital chlamydial infections and the increased risk for HIV acquisition, cost effective strategies for utilising molecular technology for the detection of genital chlamydial infection is a top priority. Further studies to evaluate this pooling strategy are ongoing.

Table 2 Laboratory diagnosis of $C$ trachomatis infection: frequency of false negative PCR/LCR FCU specimens confirmed by corresponding positive urethral/cervical swab culture results

\begin{tabular}{|c|c|c|c|c|c|}
\hline \multicolumn{3}{|l|}{ Men: } & \multicolumn{3}{|l|}{ Women: } \\
\hline Study population & $\begin{array}{l}\text { Prevalence of infection } \\
\text { (No infected/No tested) }\end{array}$ & $\begin{array}{l}\% \text { false } \\
\text { negative* }\end{array}$ & Study population & $\begin{array}{l}\text { Prevalence of } \\
\text { infection (No } \\
\text { infected/No tested) }\end{array}$ & $\begin{array}{l}\% \text { false } \\
\text { negative* }\end{array}$ \\
\hline \multicolumn{6}{|l|}{ PCR: } \\
\hline Wisenfeld ${ }^{14}$ & $62 / 362(17 \%)$ & 13 & Quinn $^{17}$ & $60 / 525(11 \%)$ & 7 \\
\hline Jaschek $^{13}$ & $81 / 530(15 \%)$ & 5 & Pasternak $^{16}$ & $39 / 666(6 \%)$ & 15 \\
\hline Bauwens $^{12}$ & $39 / 365(11 \%)$ & 3 & & & \\
\hline Quinn $^{17}$ & $75 / 713(11 \%)$ & 12 & & & \\
\hline Toye $^{15}$ & $22 / 370(6 \%)$ & 9 & & & \\
\hline \multicolumn{6}{|l|}{ LCR: } \\
\hline Deguchi $^{21}$ & $34 / 131(26 \%)$ & 6 & van Doornum ${ }^{20}$ & 25/237 (11\%) & 8 \\
\hline van Doornum ${ }^{20}$ & $29 / 163(18 \%)$ & 14 & Buimer $^{22}$ & $66 / 602(11 \%)$ & 6 \\
\hline Chernesky $^{19}$ & $154 / 1043(15 \%)$ & 7 & Chernesky $^{18}$ & $27 / 447(6 \%)$ & 4 \\
\hline Buimer $^{22}$ & $75 / 614(12 \%)$ & 8 & & & \\
\hline
\end{tabular}

$\star \%$ calculated as number of PCR/LCR false negative results/total number infected. 
We thank Dr Don Sutherland, Director of the Bureau of HIV/AIDS and STD at the Laboratory Centre for Disease Control for helpful discussions on pooling, and Elizabeth Dillon, Maria Bobrowska, and Wendi Woods for their excellen technical assistance. This study was supported in part by a grant from The Physicians' Services Incorporated Foundation.

1 World Health Organisation. Sexually transmitted diseases. Press Release WHO/64. Geneva: WHO, 25 August 1995.

2 Washington A, Johnson RE, Sanders L Jr. Chlamydia trachomatis infection in the United States: what are they costing us? FAMA 1987;257:2070-2.

3 Scholes D, Stergachis A, Heidrich FE, et al. Prevention of pelvic inflammatory disease by screening for cervical pelvic inflammatory disease by screening for cer
chlamydial infection. $N$ Engl f Med 1996;334:1362-6.

4 Plummer FA, Simonsen JN, Cameron DW, et al. Co-factors in male-female transmission of human immunodeficiency virus type 1. F Infect Dis 1991;163:233-9.

5 Grosskurth H, Mosha F, Todd J, et al. Impact of improved treatment of sexually transmitted diseases on HIV infection in rural Tanzania: randomised controlled trial. Lancet 1995:346:530-6.

6 Lin JSL, Jones WE, Yan L, et al. Underdiagnosis of Chlamydia trachomatis infection: diagnostic limitations in patients with low-level infection. Sex Transm Dis 1992;19:259-65.

7 Talbot H, Romanowski B. Factors affecting urine EIA sensitivity in the detection of Chlamydia trachomatis in men. Genitourin Med 1994;70:101-4.

8 Peeling RW. Chlamydia trachomatis and Neisseria gonorrhoeae: pathogens in retreat? Curr Opin Infect Dis gonorrhoeae:

9 Peeling RW, Brunham RC. Molecular techniques for the laboratory identification of Chlamydia trachomatis. f Intern laboratory identification of Ch
Fed Clin Chem 1994;6:78-82.

10 Schachter J, Stamm WE, Quinn TC, et al. Ligase chain reaction to detect Chlamydia trachomatis infection of the cervix. F Clin Microbiol 1994;32:2540-3.

11 de Barbeyrac B, Pellet I, Dutilh B, et al. Evaluation of the Amplicor Chlamydia trachomatis test versus culture in genital samples in various prevalence populations. Genitourin Med 1994;70:162-6.

12 Bauwens JE, Clark AM, Loeffelholz MJ, Herman SA Stamm WE. Diagnosis of Chlamydia trachomatis urethritis in men by polymerase chain rea urine. F Clin Microbiol 1993;31:3013-6.

13 Jaschek G, Gaydos CA, Welsh L, Quinn TC. Direct detection of Chlamydia trachomatis in urine specimens from symptomatic and asymptomatic men by using a rapid polymerase chain reaction assay. $\mathcal{F}$ Clin Microbiol 1993;31 1209-12.

14 Wisenfeld HC, Uhrin M, Dixon BW, Sweet RL. Diagnosis of male Chlamydia trachomatis urethritis by polymerase chain reaction. Sex Transm Dis 1994;21:268-71.

15 Toye B, Peeling RW, Jessamine P, Claman P, Gemmill I. Diagnosis of Chlamydia trachomatis infections in asymptomatic men and women by PCR assay. 7 Clin Microbiol 1996;34:1396-400.

16 Pasternak R, Vuorinen P, Kuukankorpi A, Pitkajarvi T, Miettinen A. Detection of Chlamydia trachomatis infections in womem by Amplicor PCR: comparison of diagnostic performance with urine and cervical specimens. F Clin performance with urine

17 Quinn T, Welsh L, Lentz A, et al. Diagnosis by Amplicor PCR of Chlamydia trachomatis infection in urine samples from women and men attending sexually transmitted disease clinics. 7 Clin Microbiol 1996;34:1401-6.

18 Chernesky M, Jang D, Lee H, et al. Diagnosis of Chlamydia trachomatis infections in men and women by testing first-void urine by ligase chain reaction. $\mathcal{f}$ Clin Microbio 1994;32:2682-5.

19 Chernesky MA, Lee H, Schachter J, et al. Diagnosis of Chlamydia trachomatis urethral infection in symptomatic and asymptomatic men by testing first-void urine in a ligase chain reaction assay. F Infect Dis 1994;170:1308-11.

20 van Doornum GJJ, Buimer M, Prins M, et al. Detection of Chlamydia trachomatis in urine samples from men and women by ligase chain reaction. f Clin Microbiol 1995;33: 2042-7.

21 Deguchi T, Yasuda M, Uno M, et al. Comparison among performances of a ligase chain reaction-based assay and two enzyme immunoassays in detecting Chlamydia trachomatis in urine specimens from men with nongonococcal urethritis. f Clin Microbiol 1996;34:1708-10.

22 Buimer M, van Doornum GJJ, Ching S, et al. Detection of Chlamydia trachomatis and Neisseria gonorrhoeae by ligase chain reaction-based assays with clinical specimen from various sites: Implications for diagnostic testing and screening. I Clin Microbiol 1996;34:2395-400.

23 Raboud JM, Sherlock C, Schechter MT, Lepine DG, O'Shaughnessy MV. Combining pooling and alternative (1993; 31:2298-302.

24 Verkooyen RP, Luijendijk A, Huisman WM, et al. Detection of PCR inhibitors in cervical specimens by using the Amplicor Chlamydia trachomatis assay. $f$ Clin Microbiol 1996;34:3072-4. 